Dieses Dokument ist eine Zweitveröffentlichung (Postprint) /

This is a self-archiving document (accepted version):

V. Sessi, H. Mulaosmanovic, R. Hentschel, S. Pregl, T. Mikolajick, W. M. Weber

Junction Tuning by Ferroelectric Switching in Silicon Nanowire

Schottky-Barrier Field Effect Transistors

Erstveröffentlichung in / First published in:

IEEE 18th International Conference on Nanotechnology (IEEE-NANO). Cork, 2018. IEEE. ISBN 978-1-5386-5336-4

DOI: https://doi.org/10.1109/NANO.2018.8626257

Diese Version ist verfügbar / This version is available on:

https://nbn-resolving.org/urn:nbn:de:bsz:14-qucosa2-768831 


\title{
Junction tuning by ferroelectric switching in silicon nanowire Schottky-barrier field effect transistors
}

\author{
V. Sessi ${ }^{1,2}$, H. Mulaosmanovic ${ }^{3}$, R. Hentschel $^{3}$, S. Pregl ${ }^{3}$, T. Mikolajick ${ }^{1,2,3}$, and W. M. Weber ${ }^{2,3}$ \\ ${ }^{I}$ Technische Universität Dresden, Dresden, GermanyEmail: violetta.sessi@tu-dresden.de, \\ ${ }^{2}$ Center for Advancing Electronics Dresden, Dresden, Germany \\ ${ }^{3}$ Namlab gGmbH, Dresden, Germany
}

\begin{abstract}
We report on a novel silicon nanowire-based field effect transistor with integrated ferroelectric gate oxide. The concept allows tuning the carrier transport in a non-volatile approach by switching the polarization in the ferroelectric layer close to the source Schottky-junction. We interpret the results in terms of tuning the transmissibility of the Schottkyjunction for charge carriers. The experimental results provide a first step towards the integration of memory-in-logic concepts with reconfigurable nanowire transistors.
\end{abstract}

\section{INTRODUCTION}

Silicon nanowires (SiNWs) are considered amongst the most promising building blocks for future electronic devices $[1,2]$, due to their ultimate scalability in a surround gate architecture and their compatibility with existing CMOS processes. Beyond CMOS scaling, the high sensitivity to external electric fields opens the path towards enhanced device functionality that is in the focus of current research.

One route to add new functionality is the integration of switching materials to the transistors and by this merging memory functionality into computing. Recently, it has been shown that a charge trapping layer can be added to a reconfigurable field-effect transistor (RFET) [3] and realize a nonvolatile memory function integrated into the computing device [4]. However, this solution requires quite high switching voltages. Here, we show that it is possible to introduce non-volatility in SiNW FETs by means of thin ferroelectric layers based on doped hafnium oxide $\mathrm{HfO}_{2}$.

Ferroelectricity in doped $\mathrm{HfO}_{2}$ was first reported in 2011 [5]. It is argued that this property is induced by the stabilization of a non-centrosymmetric orthorhombic phase upon annealing. Different from conventional ferroelectrics like $\mathrm{Pb}\left[\mathrm{Zr}_{\mathrm{x}} \mathrm{Ti}_{1-\mathrm{x}}\right] \mathrm{O}_{3}$ which show several integration and scaling issues, $\mathrm{HfO}_{2}$ is a high-k dielectric already in use in CMOS manufacturing. When doped with suitable dopants and/or a particular fabrication procedure is applied, the ferroelectric $\mathrm{HfO}_{2}$ phase can be stabilized.

Very important for applications, $\mathrm{HfO}_{2}$-based ferroelectrics show stable ferroelectricity with high coercive fields in the $1 \mathrm{MV} / \mathrm{cm}$ range for comparatively thin films of $5 \mathrm{~nm}-30 \mathrm{~nm}$ thickness. This allows downscaling of devices and reduction of the gate-stack height. Compatibility with miniaturized metal-oxide-semiconductor FETs (MOSFETs) has been established for laterally scaled ferroelectric FETs (FeFETs) having the channel length and width of $30 \mathrm{~nm}$ and $80 \mathrm{~nm}$, respectively [6]. Recently, large FeFET memory arrays based on $\mathrm{Si}: \mathrm{HfO}_{2}$ have been successfully demonstrated $[7,8]$.

In this work we show first results of the integration of a $\mathrm{HfO}_{2}$-based ferroelectric gate oxide in SiNW Schottky-barrier FETs (SiNW SB-FETs). Schottky contact geometries of SiNW SB-FETs provide sharp, exposed junctions whose transmissibilities can be modified by locally applied electric fields [9]. Here, we expose the injecting source junction to a $\mathrm{Al}: \mathrm{HfO}_{2}$ layer and observe non-volatile gating of the Schottky contact, which can be attributed to the remanent ferroelectric $\mathrm{Al}: \mathrm{HfO}_{2}$ polarization. The switchable ferroelectric unit functions as a non-volatile valve which either passes or blocks low energy holes. As a result, the SiNW SB-FET can be reversibly tuned by voltage pulses between two states with resistance differing by more than one order of magnitude.

\section{RESULTS AND DISCUSSION}

\section{A. SiNW SB-FET with Pt contacts}

For simplicity we build our device demonstrators with bottom-up grown SiNWs. The SiNWs are formed by the vapor-liquid-solid growth mechanism in a chemical vapor deposition (CVD) furnace, using synthesized catalytic gold nanoparticles with $20 \mathrm{~nm}$ average diameter. The gas precursor is mono-silane $\mathrm{SiH}_{4}$ and the CVD process temperature and deposition time are $\mathrm{T}=450{ }^{\circ} \mathrm{C}$ and $\mathrm{t}=40$ minutes, respectively. The resulting NWs are undoped and crystalline, with length of approx. $30 \mu \mathrm{m}$ and average diameter $20 \mathrm{~nm}$.

To provide multiple conduction channels a parallel arrangement of SiNWs is transferred to a degenerately pdoped $\mathrm{Si} / 400 \mathrm{~nm} \mathrm{SiO}_{2}$ substrate by contact printing [10]. Gold nanoparticles are chemically etched by Aqua Regia. Next, a thermal oxide is grown on the NWs by rapid thermal oxidation $\left(\mathrm{O}_{2}\right.$ at $875^{\circ} \mathrm{C}$, for 5 minutes), followed by a forming gas anneal. The resulting oxide shell is approx. $10 \mathrm{~nm}$ thick.

In this work we focus on p-type SiNW SB-FET. Thereto, the SiNWs are contacted by interdigitated Pt source (S) and drain (D) contact leads [Figs. 1 (a) and (b)] and annealed at $\mathrm{T}=500{ }^{\circ} \mathrm{C}$ for 1 minute. Pt intrusion into the SiNWs and formation of the metallic $\mathrm{Si}_{\mathrm{x}} \mathrm{Pt}_{\mathrm{y}}$ segment visible in Fig. 1 (c) deliver abrupt and exposed Schottky-junctions (SJs). The 

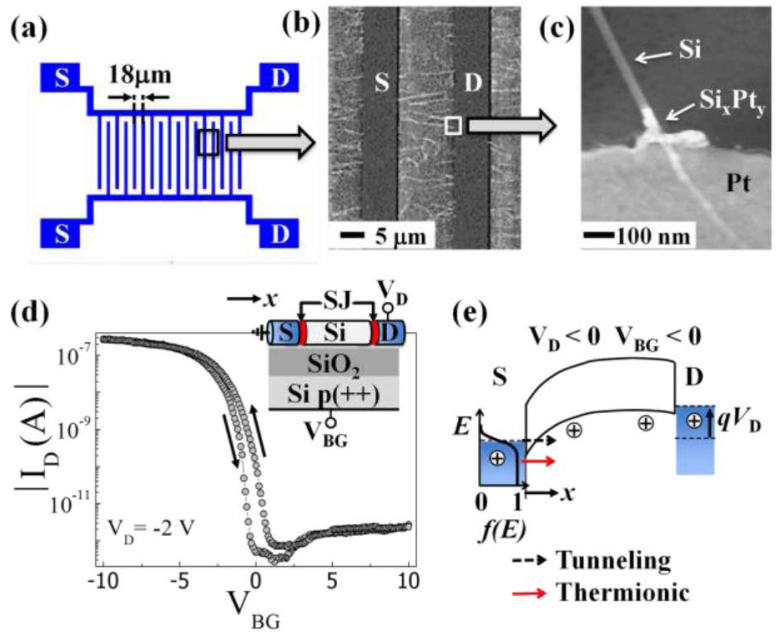

Fig. 1. SiNW SB-FET before $\mathrm{Al}: \mathrm{HfO}_{2}$ deposition. (a) Scheme of the interdigitated source (S) and drain (D) contacts. (b)-(c) Scanning electron micrograph (SEM) images showing (b) multiple NWs and (c) the interface region between $\mathrm{Si}$ and $\mathrm{Si}_{\mathrm{x}} \mathrm{Pt}_{\mathrm{y}}$. (d) Drain current $\left(\mathrm{I}_{\mathrm{D}}\right.$ ) versus back-gate voltage $\left(\mathrm{V}_{\mathrm{BG}}\right)$ for one representative device. The arrows show the sweep direction. (e) Band-diagram of the SiNW SB-FET.

residual intrinsic Si channel together with the two SJs defines a transistor with an active length of approx. $10 \mu \mathrm{m}$ along the NW axis. More details on sample fabrication can be found in Refs. [11, 12].

In Fig. 1 (d) the absolute value of the drain current $\left|I_{D}\right|$ (in the following, $\mathrm{I}_{\mathrm{D}}$ ) of the SiNW SB-FET versus the back-gate voltage $V_{B G}$ with an applied drain voltage $V_{D}=-2 \mathrm{~V}$ is shown. The predominant p-type characteristics are due to the Pt contacts, as Pt has a high work function and its Fermi level aligns near the valence band of Si. Electron transport in these devices is only achieved for high values of $\mathrm{V}_{\mathrm{BG}}$. A sketch of the band diagram can be found in Fig. 1 (e). Transport of holes across the barrier occurs via both tunneling and thermionic emission processes. The former is the main contribution to $\mathrm{I}_{\mathrm{D}}$.

\section{B. MIM structures}

Several dopants are known to induce ferroelectricity in $\mathrm{HfO}_{2}$. Examples are Si, Al, Y, Sr, La and Gd [13, 14]. Here we focus on $\mathrm{Al}$ doping, because of the availability as a standard ALD process.

Control structures made of planar metal-insulator-metal (MIM) capacitors processed on Si wafers are fabricated and characterized to determine the optimal Al concentration for integration in the NW FETs. Al: $\mathrm{HfO}_{2}$, films are deposited by atomic layer deposition (ALD) with tetrakis(ethylmethylamino)hafnium (TEMAHf), and trimethylaluminium (TMA) as precursors. The oxidant is $\mathrm{H}_{2} \mathrm{O}$ and the purge and carrier gas is $\mathrm{N}_{2}$.

The $\mathrm{Al}$ concentration is varied by the cycle ratio of the metal precursors and ranges between 2 and 16 cationic percent (cat\%). The thickness is kept constant for all samples by adjusting the number of TEMAHf:TMA supercycles.

TiN electrodes are deposited by reactive sputtering of $\mathrm{Ti}$ in $\mathrm{N}_{2}$ plasma at room temperature. The obtained MIM stacks with $20 \mathrm{~nm}$ thickness are then annealed in a rapid thermal
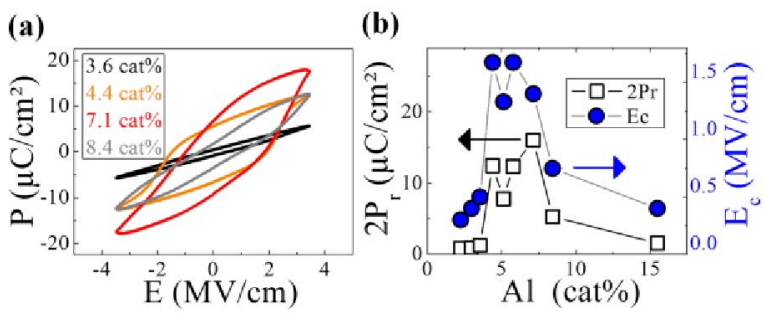

Fig. 2. MIM control structures with various $\mathrm{Al}: \mathrm{HfO}_{2}$ with different $\mathrm{Al}$ content. (a) Polarization (P) versus electric field (E) curves after $10^{4}$ field cycles. (b) Double remanent polarization $\left(2 \mathrm{P}_{\mathrm{r}}\right)$ and coercive field $\left(\mathrm{E}_{\mathrm{c}}\right)$ versus $\mathrm{Al}$ content, extracted from (a).

anneal chamber in $\mathrm{N}_{2}$ atmosphere at $800{ }^{\circ} \mathrm{C}$ to promote the ferroelectric phase. Pt dots are finally deposited on the MIM stack and provide a hard mask to structure the TiN top electrode by an $\mathrm{SC} 1$ etch.

Polarization-voltage (P-V) measurements in Fig. 2 (a) are performed by an aixACCt Systems TF Analyzer 3000. The curves are recorded after $10^{4}$ wake-up cycles, at a frequency of $10 \mathrm{kHz}$. The extracted double remanent polarization $2 \mathrm{P}_{\mathrm{r}}$ and coercive field $\mathrm{E}_{\mathrm{c}}$ values are plotted in Fig. 2 (b) versus the $\mathrm{Al}$ concentration. From these results the optimal $\mathrm{Al}$ content delivering the highest $2 \mathrm{P}_{\mathrm{r}}$ and $\mathrm{E}_{\mathrm{c}}$ values is found to be between 4 cat $\%$ and 8 cat $\%$. The reported values for $2 \mathrm{P}_{\mathrm{r}}$ and $\mathrm{E}_{\mathrm{c}}$ are comparable to what was found in previous works [15].

\section{Polarization switching effects in SiNW SB-FETs}

The Al content selected for the NW FETs is ca. $7.1 \%$, as given by 8 supercycles with (24:1) TEMAHf:TMA cycle ratio. The same layer thickness as in the MIM structures, i.e. $20 \mathrm{~nm}$, is chosen for the ferroelectric gate. Meander-shape top gates source-gate (SG) and drain-gate (DG) are structured on the sample as in Fig. 3 (a), aligned to the underlying $S$ and D contacts, respectively. The individual gates couple to the $\mathrm{S}$ and D junctions independently. Herewith it is possible to locate a specific ferroelectric polarization at a distinct device region.

The metal top gate is TiN/Pt $(10 \mathrm{~nm} / 20 \mathrm{~nm})$. Annealing of the entire gate stack is performed at reduced thermal budget ( $\mathrm{T}=600{ }^{\circ} \mathrm{C}$ for 20 seconds, in $\mathrm{N}_{2}$ atmosphere) compared to the MIM structures to avoid critical diffusion of Pt. Characterization of $\mathrm{Al}: \mathrm{HfO}_{2}$ directly on the $\mathrm{NW}$ device is shown in Figs. 3 (c)-(d). P-V measurements are performed in a shielded probe station equipped for pulsed and DC measurements. Field-cycling is performed by application of the voltage between the SG and the S, with the DG floating (see sketch in Fig. 3 (b)). The effect of wake-up cycles, also observed for the corresponding MIM structures (not shown), is visible in Fig. 3(c). After field cycling the two separated current peaks merge into a single peak and the hysteresis becomes wider, as expected for $\mathrm{HfO}_{2}$-based ferroelectrics [16]. The normalization used in Fig. 3 (d) includes the overlap area between S and SG. Compared to the MIM structures the $2 \mathrm{P}_{\mathrm{r}}$ is reduced, due to the lower annealing temperature [15]. Furthermore, the leakage increases. 
(a)

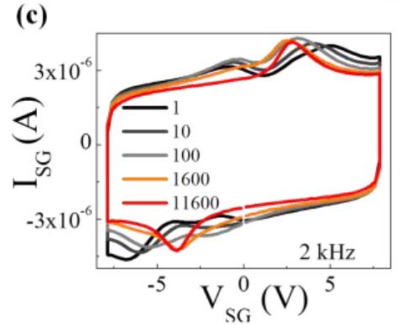

(b)

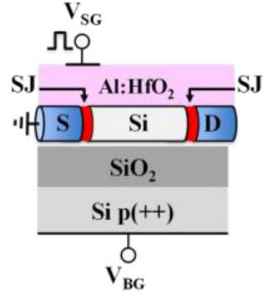

(d)

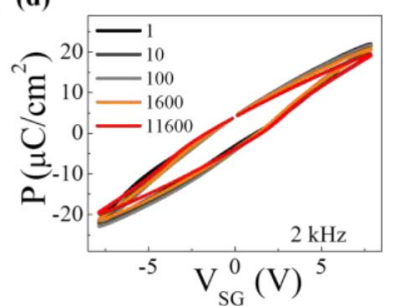

Fig. 3. SiNW SB-FET with $\mathrm{Al}: \mathrm{HfO}_{2}$ gate oxide. (a) Layout of the meander shape top gates SG and DG aligned to the S and D contacts, and SEM image of the area marked in red. (b) Measurement geometry. (c) SG Current $\left(\mathrm{I}_{\mathrm{SG}}\right)$ versus applied $\mathrm{SG}$ voltage $\left(\mathrm{V}_{\mathrm{SG}}\right)$. Each curve is recorded after the number of wake-up cycles indicated in the caption. (d) Polarization $(\mathrm{P})$ versus applied $\mathrm{V}_{\mathrm{SG}}$, extracted from (b).

However, the current peaks shown in Fig. 3 (c) clearly demonstrate ferroelectric switching.

The transfer characteristics of a SiNW SB-FET with $\mathrm{Al}: \mathrm{HfO}_{2}$ gate oxide are measured according to the geometry and sequence sketched in Figs. 4 (a) and 4 (c). A pulsed voltage $\mathrm{V}_{\mathrm{SG}}$ with amplitude of $7 \mathrm{~V}$ is applied at the $\mathrm{SG}$, as shown in Fig. 3 (a). DC-sensing is instead done by sweeping the $\mathrm{V}_{\mathrm{BG}}$ in remanence, directly after applying the pulse at $\mathrm{SG}$. In this way, we avoid a possible perturbation of the polarization state that could occur upon sweeping $\mathrm{V}_{\mathrm{SG}}$.

In Fig. 4 (c) the DC transfer characteristics of the SiNW SB FET versus $V_{B G}$ with an applied drain voltage $V_{D}=-2 V$ are shown after a positive (red, open circles) or a negative (blue, filled squares) pulse at SG. The different overlaying curves show repeated pulse/DC sensing sequences, as sketched on the top of Fig. 4 (c). When we compare the curves taken with different polarity we observe two effects: i) a sizeable shift of the threshold voltage towards more negative (positive) values after positive (negative) pulse, as expected for FETs with ferroelectric gate (see for example Ref. [17] for a p-MOSFET) and (ii) a strong reduction of the 'ON' $I_{D}$ current after positive pulse, observed for both forward and backward (not shown) scan directions. The second effect is not reported for MOSFETs, and the origin has to be sought in the distinctive energy landscape of the SiNW SB-FET. As sketched in the band diagram of Fig. 1 (e), with $\mathrm{V}_{\mathrm{D}}<0 \mathrm{~V}$ and $\mathrm{V}_{\mathrm{BG}}<0 \mathrm{~V}$ the $\mathrm{SB}$ for holes at the $\mathrm{S}$ and $\mathrm{D}$ contacts is thinned. Holes can enter the Si channel from the $\mathrm{S}$ side via both thermionic and tunneling emission. The holes flow until they leave the channel from the D side. In the specific geometry of Fig. 4 (b), a pulse generates a remanent polarization $\mathrm{P}$ in the ferroelectric domains located near the $\mathrm{SJ}$ at the $\mathrm{S}$ contact. According to our interpretation this additional, local electric field alters the shape of the potential at the $\mathrm{S}$ side, enhancing (positive pulse) or decreasing (negative pulse) the $\mathrm{SB}$, therefore effectively tuning the potential landscape of the SiNW SB-FET. As a result, blocking (positive pulse) or promoting (negative pulse) the tunneling and the thermionic emission current at the $\mathrm{S}$ contact is achieved, as schematically depicted in Fig. 4 (d)(e). In the specific case, the ferroelectric unit functions as a non-volatile valve which either passes (Fig. 4 (e)) or blocks (Fig. 4 (d)) low energy holes.

The reported effect is also observed in the DC output characteristics of Fig. 5, recorded in the ' $\mathrm{ON}$ ' state $\left(\mathrm{V}_{\mathrm{BG}}=-\right.$ $5 \mathrm{~V}$ ) after positive and negative $\mathrm{V}_{\mathrm{SG}}$ pulses. After a negative $\mathrm{V}_{\mathrm{SG}}$ pulse a non-linear output characteristics is observed, especially pronounced at low $\left|V_{D}\right|$. This behavior is typical for SB-FETs since the tunnel barrier width is decreased by $V_{D}$, with consequent non-linear enhancement of the tunneling current [11]. On the other hand after a positive $V_{S G}$ pulse, $I_{D}$ is found strongly reduced, over the entire $V_{D}$ range. It appears that in this case the influence of $V_{D}$ over the SBs is hindered, in connection with the diminished transmissibility of the $\mathrm{SJ}$ at the S contact (Fig. 4 (d)).

The device 'ON' resistance, including channel and contacts contributions, is extracted as

$$
R=\left.\left(\frac{d I_{\mathrm{D}}}{d V_{\mathrm{D}}}\right)^{-1}\right|_{\substack{V_{\mathrm{D}}=0 \mathrm{~V} \\ V_{\mathrm{BG}}=-5 \mathrm{~V}}}
$$

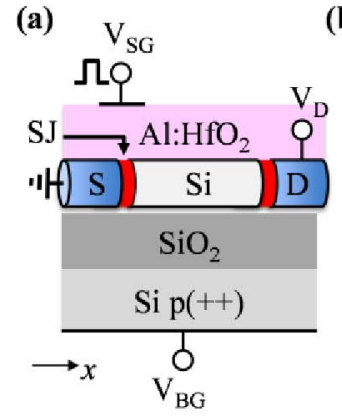

(b)
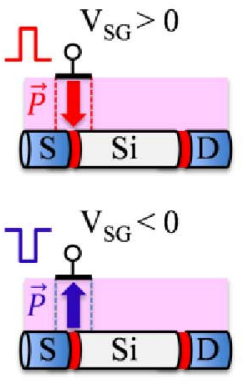

(c)

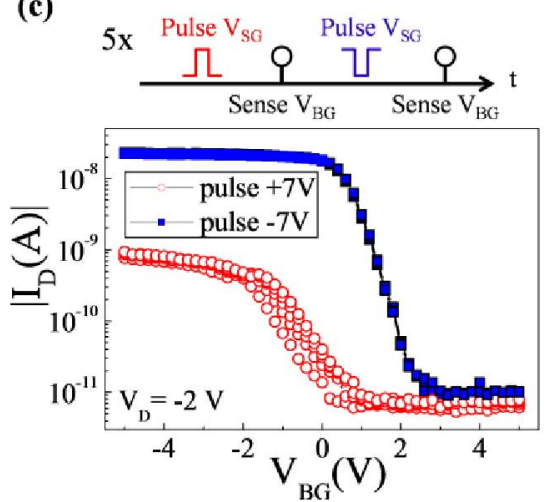

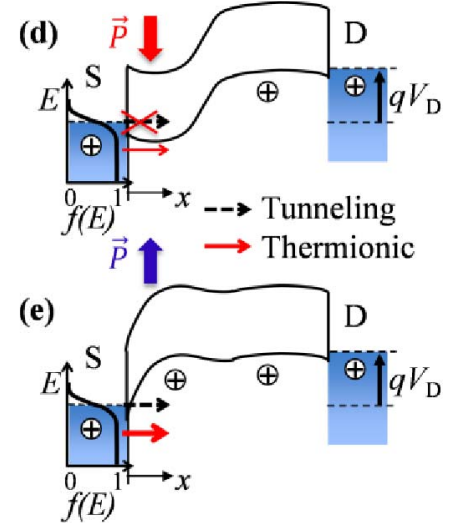

Fig. 4. Transfer characteristics of the SiNW SB-FET with $\mathrm{Al}: \mathrm{HfO}_{2}$ gate oxide. (a) Measurement geometry. (b) Generation of remanent polarization (P) near the $\mathrm{SJ}$ at the $\mathrm{S}$ contact, after positive or negative source-gate voltage pulses $\left(\mathrm{V}_{\mathrm{SG}}\right)$. (c) Drain current $\left(\mathrm{I}_{\mathrm{D}}\right)$ versus back gate voltage $\left(\mathrm{V}_{\mathrm{BG}}\right)$ after positive (red circles) and negative (blue squares) $V_{S G}$ pulses according to the sequence on top of the figure. For simplicity only the forward scans are reported. (d)(e) Sketch of the SiNW SB-FET band diagram with $\mathrm{V}_{\mathrm{D}}<0 \mathrm{~V}$ and $\mathrm{V}_{\mathrm{BG}}<0 \mathrm{~V}$, in presence of a remanent polarization near the $\mathrm{SJ}$ at the $\mathrm{S}$ contact. 


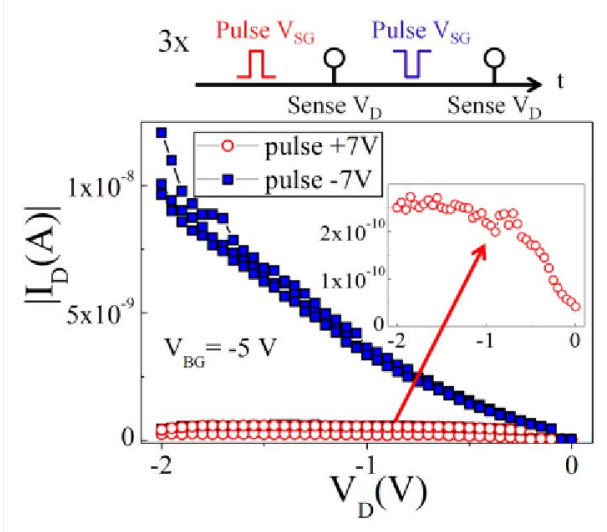

Fig. 5. Drain current $\left(I_{D}\right)$ versus drain voltage $\left(V_{D}\right)$ of the SiNW SBFET with $\mathrm{Al}: \mathrm{HfO}_{2}$ gate oxide after positive (red circles) and negative (blue squares) $V_{\mathrm{SG}}$ pulses according to the scheme on top of the figure. Inset: magnification of the output characteristic after positive $V_{S G}$ pulse.

The ratio $r$ between $R$ values calculated from (1) after positive and negative $\mathrm{V}_{\mathrm{SG}}$ pulses can be used as an efficiency parameter for non-volatile tuning of the SJ transmissibility. In the device reported here we find $r=20$.

Finally, charge trapping as a possible interpretation for the observed modification of the transistor characteristics after pulsing can be ruled out. In this case we would expect an opposite effect, e.g. a lowered $\mathrm{I}_{\mathrm{D}}$ after negative voltage pulses, as consequence of holes being trapped. Such trapping is for example responsible for the hysteresis in the transfer characteristics of Fig. 1 (d).

\section{CONCLUSION}

In conclusion, we have presented a new type of device that combines a SiNW Schottky-barrier FET with a $\mathrm{HfO}_{2}$-based ferroelectric gate stack. The Schottky junction at the source side provides a tuning knob for carrier transmissibility that can be accessed reversibly by a voltage pulse.

The achieved efficient non-volatile tuning of the Schottkybarrier properties via voltage pulses in this prototype can be of significant interest for the research community in the field of low power consumption architectures and non-volatile reconfigurability.

\section{ACKNOWLEDGMENT}

The authors gratefully acknowledge the Center for Advancing Electronics Dresden (CfAED) and the DFG project ReproNano III (WE 4853/1-3) for financial support.

\section{REFERENCES}

[1] L. J. Chen, "Silicon nanowires: the key building block for future electronic devices", J. Mater. Chem., 17, pp. 4639-4643 (2007).

[2] W. M. Weber and T. Mikolajick, "Silicon and germanium nanowire electronics: physics of conventional and unconventional transistors", Rep. Prog. Phys., 80, 066502 (2017).
[3] T. Mikolajick, A. Heinzig, J. Trommer, T. Baldauf, and W. M. Weber, "The RFET - a reconfigurable nanowire transistor and its application to novel electronic circuits and systems", Semicond. Sci. Technol., 32, 043001 (2017).

[4] S. J. Park, D. Y. Jeon, S. Piontek, M. Grube, J. Ocker, V. Sessi, A. Heinzig, J. Trommer, G. T. Kim, T. Mikolajick, and W. M. Weber, "Reconfigurable Si Nanowire Nonvolatile Transistors", Advanced Electronic Materials, 4, 1 (2018).

[5] T. S. Böscke, J. Müller, D. Bräuhaus, U. Schröder, and U. Böttger, "Ferroelectricity in hafnium oxide thin films", Appl. Phys. Lett., 99, 102903 (2011).

[6] H. Mulaosmanovic, J. Ocker, S. Müller, U. Schroede, J. Müller, P. Polakowsk, S. Flachowsky, Ra. V. Bentum, T. Mikolajick, and S. Slesazeck, "Switching Kinetics in Nanoscale Hafnium Oxide Based Ferroelectric Field-Effect Transistors", ACS Applied Materials \& Interfaces, 9, 4, pp. 3792-3798 (2017).

[7] M. Trentzsch, S. Flachowsky, R. Richter, J. Paul, B. Reimer, D. Utess, S. Jansen, H. Mulaosmanovic, S. Müller, S. Slesazeck, J. Ocker, M. Noack, J. Müller, P. Polakowski, J. Schreiter, S. Beyer, T. Mikolajick, B. Rice, "A 28nm HKMG super low power embedded NVM technology based on ferroelectric FETs", International Electron Devices Meeting (IEDM) Proceeding, pp. 11.5.1-11.5.4 (2016).

[8] S. Dünkel, M. Trentzsch, R. Richter, P. Moll, C. Fuchs, O. Gehring, M. Majer, S. Wittek, B. Müller, T. Melde1, H. Mulaosmanovic, S. Slesazeck, S. Müller, J. Ocker, M. Noack, D.-A. Löhr, P. Polakowski, J. Müller, T. Mikolajick, J. Höntschel, B. Rice, J. Pellerin, and S. Beyer, "A FeFET based super-low-power ultra-fast embedded NVM technology for 22nm FDSOI and beyond", International Electron Devices Meeting (IEDM) Proceedings, pp. 19.7.1-19.7.4 (2017).

[9] A. Heinzig, S. Slesazeck, F. Kreupl, T. Mikolajick, and Walter M. Weber, "Reconfigurable Silicon Nanowire Transistors", Nano Letters 12 (1), 119-124 (2012).

[10] S. Pregl, W. M. Weber, D. Nozaki, J. Kunstmann, L. Baraban, J. Opitz, T. Mikolajick, and G. Cuniberti, ,"Parallel arrays of Schottky barrier nanowire field effect transistors: Nanoscopic effects for macroscopic current output', Nano Research 6, 381 (2013).

[11] S. Pregl, A. Heinzig, L. Baraban, G. Cuniberti, T. Mikolajick, , and W. M. Weber, "Printable Parallel Arrays of Si Nanowire Schottky-BarrierFETs With Tunable Polarity for Complementary Logic", IEEE Transactions On Nanotechnology, 15, 549 (2016).

[12] S. Pregl, L. Baraban, V. Sessi, T. Mikolajick, W. M. Weber, and G. Cuniberti, "Signal and Noise of Schottky-Junction Parallel Silicon Nanowire Transducers for Biochemical Sensing", IEEE Sensors Journal, 18, pp. 967-975 (2018).

[13] M. H. Park, T. Schenk, C. M. Fancher, E. D. Grimley, C. Zhou, C. Richter, J. M. LeBeau, J. L. Jones, T. Mikolajick, and U. Schroeder, "A comprehensive study on the structural evolution of HfO2 thin films doped with various dopants", J. Mater. Chem. C, 5, pp. 4677-4690 (2017).

[14] U. Schroeder, E. Yurchuk, J. Müller, D. Martin, T. Schenk, P. Polakowski, C. Adelmann, M. I. Popovici, S. V. Kalinin, and T. Mikolajick, "Impact of different dopants on the switching properties of ferroelectric hafniumoxide", Japanese Journal of Applied Physics 53, 08LE02 (2014).

[15] S. Mueller, J. Mueller, A. Singh , S. Riedel , J. Sundqvist, U. Schroeder , and T. Mikolajick, "Incipient Ferroelectricity in Al-Doped $\mathrm{HfO}_{2}$ Thin Films", Advanced Functiona Materials 22, 2412-2417 (2012).

[16] T.Schenk, U. Schroeder, M. Pesič, M. Popovici, Y.V. Pershin, and T. Mikolajick, "Electric Field Cycling Behavior of Ferroelectric Hafnium Oxide", ACS Applied Materials \& Interfaces, 6, pp. 19744-19751 (2014).

[17] C.-H. Cheng and A. Chin, "Low-Leakage-Current DRAM-Like Memory Using a One-Transistor Ferroelectric MOSFET With a HfBased Gate Dielectric', IEEE Electron Device Letters, 35, pp. 07413106 (2013). 